\title{
Shoulder dystocia: incidence, mechanisms, and management strategies
}

This article was published in the following Dove Press journal:

International Journal of Women's Health

\author{
Savas Menticoglou \\ Division of Maternal-Fetal Medicine, \\ Department of Obstetrics, \\ Gynecology and Reproductive \\ Sciences, University of Manitoba, \\ Winnipeg, Canada
}

\begin{abstract}
Shoulder dystocia can lead to death or brain damage for the baby. Traction on the head can damage the brachial plexus. The diagnosis should be made when the mother cannot push the shoulders out with her own efforts with the next contraction after delivery of the head. There should be no traction on the head to diagnose shoulder dystocia. McRoberts' position is acceptable but it should not be accompanied by any traction on the head. If the posterior shoulder is in the sacral hollow then the best approach is to use posterior axillary traction to deliver the posterior shoulder and arm. If both shoulders are above the pelvic brim, the posterior arm should be brought down with Jacquemier's maneuver. If that fails, cephalic replacement or symphysiotomy is the next step. After shoulder dystocia is resolved, one should wait 1 minute or so to allow placental blood to return to the baby before cutting the umbilical cord.
\end{abstract}

Keywords: shoulder dystocia, brachial plexus injury, symphysiotomy, neonatal resuscitation, Jacquemier's maneuver

\section{Definition and incidence}

Shoulder dystocia is fundamentally a mechanical problem. The average gynecoid pelvis at the inlet is $12 \mathrm{~cm}$ in the anteroposterior diameter and $13 \mathrm{~cm}$ in the transverse diameter. The average sized fetus has a biacromial diameter of about $12-15 \mathrm{~cm} .{ }^{1,2}$ The shoulders are usually in the anteroposterior diameter above the pelvic inlet but they traverse the pelvic inlet in the larger transverse diameter. ${ }^{3}$ Although the biacromial diameter is often larger than the transverse diameter of the pelvic inlet, there is usually no obstruction because the shoulders are compressible and they are pushed forward toward the fetal chest. It can be a tight fit. ${ }^{4}$

By the time the head has exited the vulva, the fetal shoulders have usually traversed the pelvic inlet. About $20 \%$ of the time the shoulders are still in the anteroposterior diameter or slightly obliquely and will not traverse the inlet until the next uterine contraction, coincident with maternal pushing. ${ }^{3}$ If one believes that the shoulders must follow immediately after the head and thereby starts exerting downward traction on the head, one may interfere with the rotation of the shoulders at the inlet and contribute to an iatrogenic shoulder dystocia. At other times, there is a genuine mechanical impediment to delivery. If the chest circumference is too big and the biacromial diameter is, say, 16 or $17 \mathrm{~cm}$ the shoulders may not be able to enter even a normal sized pelvis. Most cases of shoulder dystocia are, indeed, caused by a big fetus trying to get through a normal sized pelvis. A few instances occur when an average sized fetus is trying to get through a small pelvic inlet. One can appreciate how a 3,700 g fetus with a biacromial diameter of $15 \mathrm{~cm}$ may have trouble getting the shoulders through a pelvic inlet of $11 \times 12 \mathrm{~cm}$. 
Shoulder dystocia is a subjective diagnosis. The only objective definition that has been proposed is a head-to-body delivery interval of more than 60 seconds. ${ }^{5}$ By this definition, some $10 \%$ of vaginal cephalic deliveries would be considered as having shoulder dystocia. This definition makes little sense, especially for those deliverers who prefer to wait for the next contraction after delivery of the head to get the shoulders delivered. The American ${ }^{6}$ and British ${ }^{7}$ Colleges of Obstetrics and Gynecology define shoulder dystocia as the failure of the shoulders to deliver with maternal pushing and gentle downward traction on the head. One problem with this definition is that you often have the mother pushing in the absence of a contraction and the second is that it implies that pulling on the head is allowed and is a normal feature of delivery. I will address this when discussing the topic of brachial plexus injury without shoulder dystocia. The definition that I prefer, but it is in the minority, is the failure of the mother to deliver the shoulders with her own maternal effort during the next contraction after the contraction that has delivered the head.

The reported incidence of shoulder dystocia is very variable, but seems to have increased in the last several decades. Whereas in the mid-20th century the usual reported incidence was $0.5 \%$ or less, it is now usually reported in the $2 \%-3 \%$ range, as high as $5 \%$ range, ${ }^{8}$ and even $>10 \%$ if one uses the head-to-body delivery interval. Part of this increased incidence is certainly due to the fact that there are more big babies being born than in the past. One speculation is that the increased use of epidural anesthesia and the practice of having women deliver on their backs might be responsible. ${ }^{9}$ It is certainly plausible that a woman unencumbered by epidural anesthesia and allowed to assume her own position for pushing would probably be able to push the shoulders out more easily. In any event, in round figures, the likelihood of encountering shoulder dystocia after a vaginal cephalic delivery is about $1 \%$ for babies weighing under $4 \mathrm{~kg}$, about $5 \%$ for babies weighing between 4 and $4.5 \mathrm{~kg}$, and about $10 \%$ for babies weighing more than $4.5 \mathrm{~kg}$.

\section{Consequences of shoulder dystocia}

Shoulder dystocia is a serious emergency for the baby. Once the head has delivered and one has the usual type of shoulder dystocia where the posterior shoulder is below the sacral promontory, the baby's chest is compressed. Although the nose and mouth are free, the baby cannot breathe because the chest is compressed. The uterus may still be contracting and interfering with blood supply to the placenta. If there is some umbilical cord compression, hypoxemia and acidosis may start to develop. If there is partial umbilical cord compression, the fetal heart may continue to pump blood through the less compressible umbilical arteries into the placenta, but compression of the more easily compressed umbilical vein may not allow blood to return from the placenta back to the fetus. Along the same lines, the increased intrathoracic pressure may not allow filling of the fetal heart. Barnum ${ }^{10}$ hypothesized this as a cause of infant death due to shoulder dystocia and the hypothesis has been proposed ${ }^{11}$ that hypovolemic shock may be the reason why some babies are unresuscitatable after shoulder dystocia. It has also been speculated that compression of the fetal neck may interfere with blood flow to and blood drainage from the brain, but this would not account for the failure of resuscitation in some cases. As a rule of thumb, once one gets to the 5-minute mark or so with unresolved shoulder dystocia there is a real chance of death or brain damage to the baby and one may have to think of extraordinary measures to resolve the shoulder dystocia. There are also implications for how to conduct the resuscitation of the baby, which will be discussed later.

Whereas death and brain damage are a consequence of the shoulder dystocia itself, the other less grave but still serious complication of brachial plexus injury is almost always a consequence of the accoucheur trying to resolve the shoulder dystocia. I am skeptical that permanent brachial plexus injury can occur without some imposed traction. I acknowledge that when the head is delivered and one or both shoulders are trapped at the pelvic brim that the neck and brachial plexus nerves may be under more stretch than usual. But the idea that the shoulders are caught up by the bony pelvis and that the uterine contractions and maternal pushing efforts continue to push the head forward while the shoulders are trapped or, in other words, the head keeps moving while the shoulders are stuck - the head is pulling the body - is too fanciful for me. It was clearly shown more than a century ago with cadaver experiments that traction on the baby's head in a direction away from the trapped shoulder makes the brachial plexus nerves taut like violin strings and that it does not take much force to have them snap. ${ }^{12,13}$ It is true that just because traction is the main cause of permanent brachial plexus injury it does not mean that all brachial plexus injuries are caused by traction, but the vast majority are.

There are three lines of evidence that are proposed as supporting the idea that brachial plexus injury may be a nontraction injury. The first is the occurrence of brachial plexus injury without described shoulder dystocia. I present one of the complications in my early career that might explain this phenomenon. 
The mother had type 1 diabetes. The head delivered easily. In anticipation of possible shoulder dystocia, the legs had been brought into McRoberts' position. After the head was delivered, I put my hands on the sides of the head and asked the mother to push and I pulled down while the patient pushed. The shoulders delivered easily. The baby weighed just over $4 \mathrm{~kg}$. As far as I was concerned, there had not been any shoulder dystocia because there had been no difficulty delivering the shoulders. When I went to make rounds the next morning, I found to my chagrin that the baby had a brachial plexus injury in the arm that had been anterior. The injury took over a year to heal with almost, but not quite, complete return of function.

When there is a brachial plexus injury and no shoulder dystocia is recorded, it is possible that sometimes this is a deliberate omission of documentation by the obstetrician so to have the defence that this was a non-iatrogenic injury. But, more often, it is likely a case where the usual customary method of delivery of the shoulders succeeds and shoulder dystocia is not appreciated. If, for example, one requires (as the standard definitions do) that the diagnosis of shoulder dystocia be made only after gentle downward traction has failed to deliver the anterior shoulder, then, if with perceived gentle downward traction the anterior shoulder is delivered, then the diagnosis of shoulder will not be made, but the brachial plexus could be injured. This is what I suspect happened in my case.

A second line of evidence proposed in support of the nontraction theory of brachial plexus palsy is that the obstetrician genuinely did not put any more traction to deliver the head than what he had always done. I present another serious brachial plexus injury in my career.

This injury occurred in the baby of a type 2 diabetic woman. In the second stage, the maternal heart rate was being recorded instead of the fetal heart rate. When this was discovered and the fetal heart rate was checked it was severely bradycardic and a midcavity vacuum delivery was done. Shoulder dystocia was identified, which was resolved by delivery of the posterior arm, which led to a fracture of the humerus. The baby was born in a depressed condition with metabolic acidosis and had moderate encephalopathy. The baby recovered well from the encephalopathy but at 1 month of age I discovered that the baby had a brachial plexus injury of the upper arm. When there was little improvement by 1 year of age, reconstructive surgery was done but at 3 years of age there was still significant handicap. I cannot remember, and the notes do not indicate, if downward traction was made on the head before the shoulder dystocia was resolved by delivery of the posterior arm (or, possibly, after the posterior arm was delivered to deliver the anterior arm), but presumably there was. Certainly there was no downward traction out of the ordinary by the resident or myself as I would have remembered that.

Most obstetricians have been taught that after delivery of the head they should put their hands on either side of the head, ask the mother to push, and then pull gently down on the head. If shoulder dystocia is encountered they are taught to try McRoberts' position or suprapubic pressure and pull gently down again. The great majority of the time both shoulders are already through the inlet by the time the head is delivered. Since the shoulders are not stuck when the obstetrician puts his hands on the side of the head and pulls on the head, there is no impediment to the shoulders and head moving together, so no harm is done. The obstetrician gets used to pulling with a certain amount of traction and the great majority of the time no harm is done. ${ }^{9}$ But for the very infrequent case where one or both shoulders are still at the pelvic brim and the shoulders do not easily follow the head, then a customary level of traction that caused no problem in hundreds of cases beforehand may be enough (especially if the fetus is asphyxiated and has no muscle tone) to cause serious injury to the brachial plexus.

The third argument proposed in support of the nontraction etiology for brachial plexus injury is the occurrence of brachial plexus injury in the arm that was posterior. If the anterior shoulder is trapped behind the pubic symphysis and the posterior shoulder is in the sacral hollow and, instead of delivering the posterior arm and shoulder directly, one pulls on the head in an upward direction, the neck and contained posterior brachial plexus can be overstretched. This was clearly shown in a picture in an 1897 article. ${ }^{12}$ Another way to damage the posterior brachial plexus is to rotate the nonrestituted head the wrong way and then pull. ${ }^{14}$

I do not think that the problem of brachial plexus injury will be solved unless obstetricians acknowledge that traction is almost invariably the cause instead of proposing other theories to spare us medicolegal problems.

\section{Prevention of shoulder dystocia}

About half of shoulder dystocia cases occur in the $10 \%$ or so of women delivering a baby weighing $4 \mathrm{~kg}$ or more. It stands to reason that if there were fewer big babies born there would be less shoulder dystocia. The main determinant of a baby's birth weight in nondiabetic women is the weight of the mother before pregnancy and the amount of weight she gains during pregnancy. In a perfect world, overweight women would get 
to their ideal weight before conception but losing weight is hard and not likely to be widely accomplished. With respect to weight gain during pregnancy, it was common practice decades ago for the obstetrician to admonish the pregnant woman if she were gaining too much, but obstetricians nowadays are loath to be scolding their patients.

The other clear risk factor for delivering a baby more than $4 \mathrm{~kg}$ is maternal diabetes. Women with diabetes have about a $20 \%$ chance of delivering a baby more than $4 \mathrm{~kg}$. The main reason to treat diabetic women with oral hypoglycemics and/or insulin is to reduce the chance of third trimester stillbirth but, of course, treatment will also reduce the chance of macrosomia. Once a woman with diabetes has a fetus with impending macrosomia (abdominal circumference measurement of about 360 or so), delivery should be strongly considered, not so much to reduce the chance of shoulder dystocia but rather to reduce the chance of unexplained stillbirth. Some women during pregnancy do not have a degree of hyperglycemia that poses any significant risk of stillbirth, but, nevertheless, glucose-lowering treatment will reduce the chance of macrosomia and presumably shoulder dystocia. ${ }^{15}$ Whether it is desirable to treat a few dozen women with insulin to prevent one shoulder dystocia is unsettled.

With respect to prevention of shoulder dystocia, however, about $90 \%$ of cases occur in women who are not diabetic. About $50 \%$ of cases will occur in women whose baby weighs more than $4 \mathrm{~kg}$. Ultrasound estimation of fetal weight is imprecise, but likely better than clinical estimation alone. An abdominal circumference of under 360 almost never indicates a fetus over 4,500 g. An abdominal circumference of $400 \mathrm{~mm}$ has a very high likelihood that the birth weight will be over 4,500 g. Between 360 and 400, there is a lot of inaccuracy. A frequently proposed remedy to reduce the chance of shoulder dystocia and its serious complications is to perform an elective cesarean section if the estimated fetal weight is 4,500 $\mathrm{g}$ or more. In Table 1 , I have estimated what such an approach would mean for the population of the hospital that I work at.

One can see that one would have to perform some 600 extra cesarean sections to prevent one case of permanent brachial plexus injury. Of course shoulder dystocia can lead to fetal brain damage so it is possible that the 600 extra cesarean sections would also prevent the rare case of baby brain damage or death. Cesarean section is one of the safest operations in the world, but several hundred cesarean sections to prevent one bad outcome seems excessive to me although others may legitimately disagree and the mother may consider this a reasonable tradeoff. If one believes, for
Table I Estimated number of permanent BPI prevented by routine CS for fetuses greater than 4,500 grams

\begin{tabular}{|c|c|c|c|}
\hline \multicolumn{4}{|l|}{ Assumptions } \\
\hline \multicolumn{4}{|c|}{ I. 100,000 births } \\
\hline \multicolumn{4}{|c|}{ 2. 13,000 BW $4,000-4,500 \mathrm{~g} ; 15 \%$ CS in labor } \\
\hline \multicolumn{4}{|c|}{ 3. $2,000 \mathrm{BW}>4,500 \mathrm{~g} ; 30 \% \mathrm{CS}$ in labor } \\
\hline \multicolumn{4}{|c|}{ 4. $5 \% \mathrm{SD}$ in $4,000-4,500 \mathrm{~g}$ group } \\
\hline \multicolumn{4}{|c|}{$10 \%$ SD in $>4,500$ g group } \\
\hline \multicolumn{4}{|c|}{ 5. $10 \% \mathrm{BPI}$ with SD } \\
\hline \multicolumn{4}{|c|}{ 6. $20 \%$ of $\mathrm{BPI}$ are permanent } \\
\hline \multicolumn{4}{|c|}{$\begin{array}{l}\text { US will falsely identify } 20 \%(\mathrm{~N}=2,600) \text { of } 4,000-4,500 \mathrm{~g} \text { group } \\
\text { as }>4,500 \mathrm{~g}\end{array}$} \\
\hline \multicolumn{2}{|l|}{$4,000-4,500 \mathrm{~g}$} & \multicolumn{2}{|c|}{$>4,500 \mathrm{~g}$} \\
\hline \multicolumn{2}{|l|}{13,000} & \multicolumn{2}{|c|}{2,000} \\
\hline$\downarrow$ & $2,000 \mathrm{CS}$ in labor & $\downarrow$ & 600 CS in labor \\
\hline \multicolumn{2}{|l|}{ II,000 vag del } & \multicolumn{2}{|c|}{$\mathrm{I}, 400$ vag del } \\
\hline \multicolumn{2}{|l|}{$\downarrow$} & \multicolumn{2}{|l|}{$\downarrow$} \\
\hline \multicolumn{2}{|l|}{550 SD } & \multicolumn{2}{|c|}{$140 \mathrm{SD}$} \\
\hline \multicolumn{2}{|l|}{$\downarrow$} & \multicolumn{2}{|l|}{$\downarrow$} \\
\hline \multicolumn{2}{|l|}{$55 \mathrm{BPI}$} & \multicolumn{2}{|c|}{ I4 BPI } \\
\hline \multicolumn{2}{|l|}{$\downarrow$} & \multicolumn{2}{|l|}{$\downarrow$} \\
\hline \multicolumn{2}{|l|}{ II perm BPI } & \multicolumn{2}{|c|}{3 perm BPI } \\
\hline
\end{tabular}

Notes: Policy of routine CS if $>4,500 \mathrm{~g}$ results in $2,000+2,600=4,600 \mathrm{CS}$. Of these, $600+(15 \%$ of 2,600$)=1,000$ would have had a CS in labor. Therefore: 3,600 extra CS. Would have prevented $3 \mathrm{BPI}$ in $>4,500 \mathrm{~g}$ and $3 \mathrm{BPI}$ in the 2,600 women in 4,000$4,500 \mathrm{~g}$ group who avoided attempted vaginal delivery.

Abbreviations: BPI, brachial plexus injury; BW, birth weight; CS, caesarean section; Perm, permanent; SD, shoulder dystocia.

example, that all breech presenting fetuses in labor should be delivered by cesarean section then one is implicitly accepting that doing several hundred cesarean sections to prevent one bad outcome is reasonable. Similarly, in a suitably chosen woman who is attempting a trial of labor after cesarean, the chance of a major catastrophe, namely, death or damage to the baby, is about 1 in 1,000 attempts. We allow women to choose elective repeat cesarean section for such a small risk; one then has to accede to a woman's request for an elective cesarean section if the baby is thought to be too big.

One interesting attempt to try to better predict those instances where shoulder dystocia with injury might occur has been proposed. ${ }^{16}$ The model essentially takes the estimated fetal weight with the maternal weight and height and calculates a risk of shoulder dystocia with injury. In this model, to prevent one case of shoulder dystocia with brachial plexus injury, I estimated that 27 extra cesarean sections would have to be performed. If one assumes that $20 \%$ of those injuries would be permanent, it comes to 135 extra cesarean sections to prevent a permanent injury. The model is promising, but still one is doing many cesarean sections to prevent one bad outcome. 
A final approach to reducing the chance of macrosomia and shoulder dystocia is to induce labor early if the baby is thought to be getting too big. One prospective study has addressed the question of labor induction to prevent shoulder dystocia. ${ }^{17}$ Instead of waiting to induce labor when the fetus was already thought to be $4 \mathrm{~kg}$ or more, induction was carried out between 36 and 39 weeks when the fetus was thought to be big clinically and on ultrasound. The authors demonstrated that the induction of labor group had a lower cesarean section rate $(28 \%$ vs $32 \%)$ and that the rate of shoulder dystocia of any type was $4 \%$ vs $8 \%$. In the induction group, the mean birth weight was about $300 \mathrm{~g}$ less. At first look, the results are impressive. Disconcerting, however, is that despite the fact that less than half the babies weighed over $4 \mathrm{~kg}$ and $<10 \%$ were more than $4,500 \mathrm{~g}$, nevertheless, the overall cesarean section rate in the study was $30 \%$. In a study carried out in my hospital 25 years ago ${ }^{18}$ where all babies had a birthweight of $4,500 \mathrm{~g}$ or more, the cesarean section rate in women without a previous cesarean section was just over $20 \%$. The problem with ultrasound estimation of fetal weight is that if one tells the obstetrician or the pregnant woman that the fetus may be big, that knowledge by itself will bias people to perform a cesarean section, either before labor or during labor if labor is going slower than expected.

\section{Management of shoulder dystocia General considerations}

If there is worry that the fetus might be macrosomic, or that there is an otherwise increased risk of shoulder dystocia, for example, a mid-cavity delivery after a long second stage, or if the woman is obese and has diabetes, then delivery should not be in the same room where labor occurred. Delivery should be in an operating room. If serious shoulder dystocia is encountered and one has to consider a general anesthetic or complicated obstetrical maneuvers you do not want to be wasting valuable minutes going from a birthing room to an operating room.

Unless one is doing an operative vaginal delivery for the head (in which case one has no choice) the lower end of the bed should not be broken. This tempts the accoucheur to pull down on the head to deliver the shoulders. Leave the bed unbroken. One should not pull on the head at all. If the shoulders have not delivered with the same contraction as the head, I prefer to wait for the next contraction in almost all circumstances. It may take $2-3$ minutes for the next contraction to come and it is unnerving for those who have become accustomed to aiming for delivery of the shoulders right after the head. When the next contraction comes, the mother is encouraged to push strongly and it may take 3-4 pushes during the contraction for delivery of the shoulders. One should avoid pulling on the head.

One legitimate concern and an unsettled issue is the following: if there is an apprehension that shoulder dystocia may occur and if, indeed, this turns out to be a case of genuine shoulder dystocia then one has lost 2-3 valuable minutes in waiting that could have been used to try and resolve the shoulder dystocia. This is a downside of waiting for shoulders.

In certain circumstances, the delivery of the head can give clues as to whether one might be dealing with shoulder dystocia. If, after delivery of the head, with or without instruments, the face and chin are delivered with difficulty, the chin has to be hooked over the perineum, or the perineum has to be pushed back to allow the chin to deliver, or after the head has delivered the head is sucked back against the vulva and does not restitute at all, if these conditions apply, it is probably not a good idea to wait 2-3 minutes for the next contraction before asking the mother to push vigorously. If with vigorous maternal pushing the mother cannot push the shoulders out, then the diagnosis of shoulder dystocia can be made. But there should be no pulling on the baby's head.

Once the diagnosis of shoulder dystocia is anticipated or is made, extra help should be summoned, including an anesthesiologist if not already done beforehand, the bed should be broken, and the mother's buttocks brought down beyond the edge of the table, and the hips should be flexed so that the knees are close to the mother's chest. This is the so-called McRoberts' position.

The McRoberts' position has advantages. The first is that in some instances sharply flexing the mother's legs against her chest will move the symphysis pubis over the anterior shoulder helping to dislodge it. The other is that the position may allow the posterior shoulder to slide down further into the sacral hollow and make access to the posterior arm and shoulder easier. The McRoberts' position is fine; the problem is with McRoberts' maneuver. After the McRoberts' position is adopted, the usual advice is for the delivering obstetrician to now pull on the baby's head in concert with maternal pushing. Indeed, another purported advantage given for the McRoberts' maneuver is that the traction forces are less than when the mother is not in McRoberts' position. The next step described is to apply suprapubic pressure on the stuck anterior shoulder to try to dislodge the shoulder from behind the symphysis pubis. This maneuver is also followed with traction on the head. 
The guidelines of the expert bodies (the American, ${ }^{6}$ the British, ${ }^{7}$ and the French ${ }^{19}$ ) all endorse the McRoberts' maneuver with or without suprapubic pressure as the firstline treatment for the management of shoulder dystocia. I disagree. The reported success rates with these maneuvers as first-line measures is about $50 \%$, with higher success rates generally in the reports with the highest incidences of shoulder dystocia. Who can argue with this? The problem is that when one looks at reports ${ }^{20-23}$ of brachial plexus injury when the only maneuvers that have been used are the McRoberts' maneuver, with or without suprapubic pressure, there is about a $10 \%$ incidence of brachial plexus injury. The fact is that in the usual case of shoulder dystocia there have often been at least three traction efforts on the baby's head, namely, the first downward traction used to diagnose shoulder dystocia and then a second downward traction attempt with the McRoberts' maneuver, and a third traction attempt with the suprapubic pressure maneuvers.

The defence of using the McRoberts' maneuver and suprapubic pressure as first-line treatment is that they are easy to perform and that they often resolve the shoulder dystocia. The further defence is that when comparison is made with the incidence of brachial plexus injury when the other standard maneuvers are used, such as rotation of the shoulders or delivery of the posterior or anterior arm, the rate of brachial plexus injury seems to be the same. What is not emphasized, however, is that recourse to the other standard maneuvers is almost invariably made after there have been failed attempts with McRoberts' maneuver and/or suprapubic pressure. As rotation maneuvers and direct arm extraction maneuvers do not cause lateral traction on the brachial plexus, most such injuries are almost certainly a consequence of the initial downward traction used to diagnose shoulder dystocia or to the traction attempts during the McRoberts'/suprapubic combination or to upward traction on the fetal head to try to free the posterior shoulder. McRoberts' position is good; McRoberts' maneuver, if that involves traction, is bad.

A final consideration: it is imperative that one avoid cutting any nuchal cord before delivery. If shoulder dystocia occurs it is a disaster. ${ }^{24,25}$

\section{Specific maneuvers}

So the diagnosis of shoulder dystocia has been made by the inability of the mother to push the shoulders out by her own efforts and even after the McRoberts' position has been employed. What is the next step? The next step is to put one's dominant hand into the posterior part of the pelvis and determine if the posterior shoulder is in the sacral concavity or not.
Almost always, the posterior shoulder has gotten past the sacral promontory and is present somewhere along the curve of the sacrum. This will virtually always be resolvable with the standard obstetrical maneuvers. There are two standard approaches, the rotational maneuvers and the arm extraction maneuvers.

In the rotational maneuvers, the best approach is to use one's dominant hand (not just two fingers) and introduce the palm of the hand into the 6 o'clock position in the pelvis behind the shoulder, as if applying the first forceps blade in a forceps delivery, and then move that hand to the left side (for right handers) of the mother's pelvis. The hand will now be either at the anterior part of the posterior shoulder or the posterior part of the posterior shoulder. If it is the anterior surface of the posterior shoulder that one's hand is against, one will now try to rotate the posterior shoulder clockwise in order to corkscrew the shoulders through the pelvis. This is the only time when some fundal pressure may be allowed, although it has to be under the direction and coordination of the obstetrician. If it works, the posterior shoulder will rotate around so that it lies under the symphysis pubis and the previous anterior shoulder will now be in the hollow of the sacrum and delivery should proceed smoothly after that. This is the Woods screw maneuver. ${ }^{26}$

If it is the baby's left shoulder that is lying posteriorly in the sacral hollow, then when the obstetrician puts his dominant hand into the left side of the maternal pelvis, the hand will be against the posterior aspect of the posterior shoulder. Now, again, clockwise rotation attempts are made and can be synchronized with attempts to push the stuck anterior shoulder dorsally to the mother's left. This is the reverse Woods maneuver that was described by Rubin. ${ }^{27}$ One can of course try to rotate the shoulders counter clockwise, but, unless one is left handed, the left hand is not as effective in causing rotation.

If the rotation maneuvers do not work, one should proceed to try to extract one of the arms. This was the great insight of Jacquemier $^{28}$ in 1860 . The idea is that if one can extract one of the arms then one can convert the obstructing diameter from a biacromial diameter to an acromial-axillary diameter thereby reducing the biacromial width by the $2-3 \mathrm{~cm}$ width of one shoulder.

Usually, the anterior arm is too difficult to reach behind the subpubic arch with the shoulder impacted against the symphysis pubis. However, it is worth trying a Couder maneuver ${ }^{29,30}$ to see if it will work. This involves taking the index and middle fingers of one hand and, using them as a splint, try to place them on the anterior surface of the upper humerus. These two fingers now try to push the 
humerus backward toward the baby's back and this will sometimes flex the elbow and let the hand appear under the symphysis. The hand can now be grasped and the anterior arm delivered.

The usual approach is to try to deliver the posterior arm first. This was described in English by Barnum ${ }^{10}$ and is described in all of the textbooks. The operator follows the shoulder to the humerus and hopes to find the elbow and forearm of the posterior arm lying over the baby's chest and, again, one grasps the forearm and sweeps it across the baby's chest and outside the vulva. Generally once the posterior arm and shoulder have delivered, the anterior shoulder will fall beneath the symphysis pubis and be easily delivered. If the anterior shoulder does not follow, one can try a Couder maneuver again or, using the delivered posterior arm, rotate the baby so that the anterior shoulder ends up in the posterior pelvis where it can be delivered. One should not pull on the head.

When delivery of the posterior arm fails, it is because the posterior arm is not lying across the fetal chest but is lying extended under the baby's body with the forearm and hand unreachable. This is where posterior axillary traction should be used. ${ }^{31,32}$ It is my preferred approach to all cases where the posterior shoulder is in the sacral concavity. This involves not extracting the posterior arm first but delivering the posterior shoulder first. One puts the middle fingers (not index fingers - they are not strong enough) into the axilla like hooks and pull down the posterior shoulder with the overlapping fingers. Ideally an assistant is tilting (not pulling) the baby's head upward. The operator should be on his knees. One pulls the posterior shoulder down using traction with the overlapping middle fingers in the axilla. One should not pull parallel to the floor, but instead drag the posterior shoulder along the curve of the sacrum until the posterior shoulder and then arm emerge. This is by no means a novel technique. In fact, it was described in 1609 by the royal midwife Louise Bourgeois. In Jacquemier's treatise on shoulder dystocia, the technique is often described. English-language textbooks did not describe it in the past because of concern that the fingers in the posterior axilla could somehow damage the brachial plexus by direct pressure (a theory proposed by Erb), but this fear is unfounded.

On very rare occasions, the two middle fingers together cannot pull the posterior shoulder down and it is necessary to pass a strong, thin nonstretchable cord or catheter around the axilla to hook it and then pull down on the cord with a Kocher clamp. ${ }^{33}$ One can also impart rotation with this approach. Almost invariably once the posterior shoulder and arm are delivered, the anterior shoulder will release itself from the symphysis pubis. If not, one must resist the temptation to pull down on the head. Instead, use a Couder maneuver to free the anterior arm or rotate the baby by the arm $180^{\circ}$.

The apprehension that some have with delivering the posterior arm and, especially, the posterior shoulder directly is the high chance (in my experience, about one in four) of fracturing the humerus. In many articles, fractured humeri and brachial plexus injuries are lumped together as birth trauma, but the comparison makes no sense. Fractured humeri always heal. There is never permanent injury. A permanent brachial plexus injury is a serious lifelong disability.

What if shoulder dystocia is diagnosed and the vaginal hand shows that the posterior shoulder is not in the pelvis, that is, the posterior shoulder is caught up above the sacral promontory, in other words, there is a double shoulder dystocia? One has a big problem.

The classic approach is to use the Jacquemier's maneuver described in $1860 .{ }^{28}$ It is essentially trying to deliver the posterior arm, but different in some respects from when the posterior shoulder is already in the sacral hollow. Delivery of the posterior arm when the posterior shoulder is above the sacral promontory is virtually never described in Englishlanguage textbooks or articles. The best descriptions, with illustrations, are in Italian and French textbooks. ${ }^{34,35}$

The mother's hips are past the edge of the bed. The operator is on one knee in front of the patient. The hand that will be used is the one that corresponds to the baby's abdomen. If it is the baby's right shoulder that is posterior, the operator's right hand is used. If it is the baby's left shoulder that is posterior it is the operator's left hand that is used. The operator uses his gloved hand but the forearm is bare and well lubricated so as to reduce the friction of a gown when the forearm up until the elbow is inside the vagina. The operator is on one knee because the forearm has to follow the maternal axis from the coccyx to the umbilicus. Getting the forearm into the pelvis and past the sacral promontory is not difficult because the pelvis is empty apart from the baby's neck. The hand and lower forearm are passed beyond the pelvic inlet and one seeks to find the baby's forearm and bring down that arm. Delivery is generally straightforward after this. In centers experienced in the technique, it succeeds more than nine times out of ten. ${ }^{36}$

Another technique described (with which I have no personal experience) is the Letellier maneuver, ${ }^{34,35}$ which aims to deliver the posterior shoulder trapped above the sacral promontory. This time one uses the hand corresponding to the fetal back (right hand for the left shoulder, left hand for the right shoulder). One reaches upward and tries to hook the index finger into the posterior part of the axilla and the 
thumb in the anterior part of the axilla. Instead of trying to pull the posterior shoulder directly downward the posterior shoulder is pushed in the direction of the pubis while simultaneously rotating it along the pelvic brim. If one is fortunate, the posterior shoulder can be rotated under the pubis and that arm brought down.

If after 3-4 minutes the Jacquemier \pm Letellier maneuvers have failed or else one has been able to deliver the posterior arm and still the shoulder dystocia cannot be resolved, one is now faced with a catastrophic shoulder dystocia and something drastic has to be done.

The procedure most described is to try to push the already delivered head back into the vagina and proceed to do a cesarean section. The majority of the time this is described as easy and if indeed the head does enter the vagina easily and a quick cesarean section can be done then this is probably the best way to proceed. In cases where cephalic replacement has been unsuccessful, there are descriptions of doing hysterotomies and through the hysterotomy trying to dislodge the stuck anterior shoulder or else trying to pass the stuck posterior shoulder from above into the sacral hollow to be delivered by an assistant from the vagina. I have no personal experience with either method.

To me the best solution is to proceed with a symphysiotomy. Whenever one is anticipating the possibility of shoulder dystocia one should be physically and psychologically prepared to proceed with symphysiotomy. A $3 \mathrm{~cm}$ separation of the symphysis pubis will enlarge the diameter of the pelvic inlet by some $25 \%{ }^{37}$ and resolve every case of shoulder dystocia. A Foley catheter should be left in place or, alternatively, be inserted if shoulder dystocia is more than a remote possibility. Trying to insert a catheter through the urethra when the head has already delivered and the shoulders are stuck is difficult. A scalpel should be at hand. If an anesthesiologist is not already present to put the woman to sleep or if the epidural anesthetic has not been topped up, the mons veneris over the symphysis pubis and the underlying symphysis ligament should be infiltrated with xylocaine.

Rubin $^{27}$ proposed symphysiotomy as a desperate measure in his article on shoulder dystocia. There are about a dozen cases published of symphysiotomy done for shoulder dystocia. In every single case where symphysiotomy was done, there was immediate delivery of the body. However, the outcome is not uniformly good for the baby. The procedure has been described as "too little, too late" but only the "too late" part is correct. The $25 \%$ enlargement of the pelvic inlet always allows easy delivery of the shoulders. However, the procedure must be done promptly. In the three cases described in one article, ${ }^{38}$ the procedures were done
12 minutes, 13 minutes, and 23 minutes after delivery of the head, and of course all the outcomes were bad. In another report ${ }^{25}$ of two cases, there was again immediate delivery of the body, but one outcome was bad because a nuchal cord had been clamped and cut before shoulder dystocia was recognized. As has been stated in using symphysiotomy for the trapped after-coming head of a breech, waiting too long to do a symphysiotomy merely facilitates the delivery of a dead or brain-damaged baby. ${ }^{37}$ The best description of the technique, with pictures, is that of G. Mola. ${ }^{39}$ One caveat with symphysiotomy is to never let the vaginal middle finger deviate from its position under the symphysis. The urethra and, more importantly, the fetal neck are located under the symphysis.

There is no clear cut time limit as to how fast one should try to resolve shoulder dystocia but after 4-5 minutes of trying other maneuvers without success, one should be thinking about symphysiotomy. It does not fail in resolving shoulder dystocia. It always works.

The legitimate argument will be proposed that it is much better to have performed the several hundred elective cesarean sections necessary to prevent a permanent injury from shoulder dystocia for the baby rather than find oneself in the situation of having to perform a symphysiotomy. There is no doubt whatsoever that the woman recovering from a symphysiotomy will be more miserable for 2-3 weeks than the woman who has had an elective cesarean section instead. But the proper comparison is not between one symphysiotomy and one cesarean section. The proper comparison is the morbidity from one symphysiotomy vs the several hundred needless cesarean sections, let alone the subsequent repeats, to prevent one symphysiotomy. It is the same dilemma with vaginal breech delivery. To prevent one catastrophe in a vaginally born breech baby, you have to do several hundred cesarean sections. I have always felt that anybody who is going to undertake vaginal breech delivery should always be physically and psychologically prepared to perform a symphysiotomy if the need arises. The same applies to shoulder dystocia.

\section{Resuscitating the baby after shoulder dystocia}

After the obstetrician or midwife has finally succeeded in resolving a shoulder dystocia, after great stress and anxiety, and a limp, depressed baby is born, and the neonatal team is anxiously waiting to resuscitate the baby, there is an inevitable natural tendency to immediately clamp and cut the umbilical cord so as to hand the baby over to the waiting resuscitators. This is the wrong thing to do. Even if the shoulder dystocia 
has taken less than 5 minutes to resolve, some babies come out without a pulse and cannot be resuscitated. ${ }^{40}$ The problem is not lack of oxygen. Expert resuscitators should be able to resuscitate without subsequent brain damage babies born after 5-10 minutes of complete uterine rupture or abruptio placenta or sudden unexplained bradycardia. The difference with the depressed baby after shoulder dystocia is that in addition to interruption of oxygen supply, the baby may also be severely hypovolemic. ${ }^{11,41}$ While the baby's chest has been severely compressed within the pelvis, the baby's heart has been able to pump blood through the umbilical arteries into the placenta; however, because of the chest compression or umbilical vein compression, placental blood cannot return back to the chest. The worst thing when the baby comes out limp is to immediately clamp the cord. The resuscitators should come to the baby instead of the baby being taken to the resuscitators. A minute or so should be allowed for the fetal blood accumulated in the placenta to return back to the baby's circulation.

\section{Conclusion}

1. Shoulder dystocia is a serious emergency. If it is not resolved, the baby can die or be brain damaged. If one acts impetuously and pulls on the head, the brachial plexus can be permanently injured.

2. The admonition has been made "do not pull hard, do not pull quickly, and do not pull down." I believe that one should not pull AT ALL, neither to make the diagnosis of shoulder dystocia nor as part of the McRoberts' maneuver or in conjunction with suprapubic pressure.

3. If the fetus is macrosomic or the mother is obese or diabetic, delivery should not take place in a birthing room but rather in an operating room.

4. Do not clamp and cut a nuchal cord.

5. Be prepared for the worst, including the need for symphysiotomy and general anesthesia.

6. Shoulder dystocia is diagnosed when the mother cannot push the shoulders out with the subsequent contraction. If an urgent vacuum or forceps delivery has been done for severe bradycardia or a terrible tracing, or if there is a strong presumption of shoulder dystocia because the chin is tight against the vulva, do not wait until the next contraction.

7. McRoberts' position is fine; McRoberts' maneuver, pulling on the head, is not.

8. Put your hand in the vagina and find out if the posterior shoulder is in the sacral hollow or not.

9. If the posterior shoulder is in the sacral hollow, the most effective approach is to use posterior axillary traction, either with overlapping middle fingers or with a sling, and drag the shoulder along the sacral hollow until the shoulder and arm are delivered. The humerus will often fracture. This is unfortunate but not disastrous. Dozens of fractured humeri are better than one permanent brachial plexus injury with a useless arm.

10. If the posterior shoulder is not in the sacral hollow but is at the pelvic brim, the best maneuver is the Jacquemier maneuver and properly performed will almost always work.

11. If after 5 minutes or so, the shoulder dystocia has not been resolved, try to push the delivered head back into the vagina and if it goes in easily proceed to a cesarean section. If the head cannot be replaced easily, proceed to a symphysiotomy. Others would proceed to symphysiotomy instead of trying to replace the head.

12. After the baby is born, do not immediately clamp and cut the umbilical cord. Let a minute go by so that the baby can be autotransfused through the placenta and have the baby resuscitators start their resuscitating at the mother's bedside.

\section{Disclosure}

The author reports no conflict of interest in this work. The author would like to disclose that as the patients have been lost to follow-up, patient written informed consent for publication of these case details was not possible. The details have been sufficiently anonymized as not to cause harm to the patient.

\section{References}

1. Verspyck E, Goffinet F, Hellot MF, Milliez J, Marpeau L. Newborn shoulder width: a prospective study of 2222 consecutive measurements. Br J Obstet Gynaecol. 1999;106(6):589-593.

2. Youssef AEA, Amin AF, Khalaf M, Khalaf MS, Ali MK, Abbas AM. Fetal biacromial diameter as a new ultrasound measure for prediction of macrosomia in term pregnancy: a prospective observational study. J Matern Fetal Neonatal Med. 2018;7:1-6.

3. Borell U, Fernstrom I. Radiographic studies of the rotation of the fostal shoulders during labour. Acta Obstet Gynecol Scand. 1958;37(1): 54-61.

4. Sreekanth R, Thomas BP. Human evolution: the real cause for birth palsy. West Indian Med J. 2015;64(4):424-428.

5. Spong CY, Beall M, Rodrigues D, Ross MG. An objective definition of shoulder dystocia: prolonged head-to-body delivery intervals and/or the use of ancillary obstetric maneuvers. Obstet Gynecol. 1995;86(3): 433-436.

6. Committee on Practice Bulletins-Obstetrics. Practice Bulletin No 178: Shoulder dystocia. Obstet Gynecol. 2017;129(5):e123-e133.

7. Royal College of Obstetricians and Gynaecologists. Shoulder dystocia. Green-top Guideline No. 42:2012.

8. Gurewitsch Allen ED, Will SEB, Allen RH, Satin AJ. Improving shoulder dystocia management and outcomes with a targeted quality assurance program. Am J Perinatol. 2017;34(11):1088-1096.

9. Mortimore VR, McNabb M. A six-year retrospective analysis of shoulder dystocia and delivery of the shoulders. Midwifery. 1998;14(3): 162-173. 
10. Barnum CG. Dystocia due to the shoulders. Am J Obstet Gynecol. 1945; 50(4):439-442.

11. Mercer J, Erickson-Owens D, Skovgaard R. Cardiac asystole at birth: is hypovolemic shock the cause? Med Hypotheses. 2009;72(4): 458-463.

12. Fieux G. De la pathogéne des paralysis brachiales chez le nouveau-né. Paralyses obstetricales [The pathogenesis of brachial palsy in the neonate]. Ann Gynecol Obstet. 1897;47:52-64. French.

13. Clark LP, Taylor AS, Prout TP. A study on brachial birth palsy. Am J Med Sci. 1905;130(4):670-707.

14. Khunda A. Shoulder dystocia and Erb's palsy: is the accoucheur always guilty of negligence? BJOG. 2014;121(7):912.

15. Crowther CA, Hiller JE, Moss JR, et al; Australian Carbohydrate Intolerance Study in Pregnant Women (ACHOIS) Trial Group. Effect of treatment of gestational diabetes mellitus on pregnancy outcomes. N Engl J Med. 2005;352(24):2477-2486.

16. Dyachenko A, Ciampi A, Fahey J, Mighty H, Oppenheimer L, Hamilton EF. Prediction of risk for shoulder dystocia with neonatal injury. Am J Obstet Gynecol. 2006;195(6):1544-1549.

17. Boulvain M, Senat MV, Perrotin F, et al; Groupe de Recherche en Obstétrique et Gynécologie (GROG). Induction of labour versus expectant management for large-for-date fetuses: a randomised controlled trial. Lancet. 2015;385(9987):2600-2605.

18. Menticoglou SM, Manning FA, Morrison I, Harman CR. Must macrosomic fetuses be delivered by a caesarean section? A review of outcome for 786 babies greater than or equal to 4,500 g. Aust $N Z J$ Obstet Gynaecol. 1992;32(2):100-103.

19. Sentilhes L, Sénat MV, Boulogne AI, et al. Shoulder dystocia: guidelines for clinical practice from the French College of Gynecologists and Obstetricians (CNGOF). Eur J Obstet Gynecol Reprod Biol. 2016; 203:156-161

20. Leung TY, Stuart O, Suen SS, Sahota DS, Lau TK, Lao TT. Comparison of perinatal outcomes of shoulder dystocia alleviated by different type and sequence of manoeuvres: a retrospective review. BJOG. 2011;118(8):985-990.

21. Gherman RB, Ouzounian JG, Goodwin TM. Obstetric maneuvers for shoulder dystocia and associated fetal morbidity. Am J Obstet Gynecol. 1998;178(6):1126-1130.

22. McFarland MB, Langer O, Piper JM, Berkus MD. Perinatal outcome and the type and number of maneuvers in shoulder dystocia. Int J Gynaecol Obstet. 1996;55(3):219-224.

23. Spain JE, Frey HA, Tuuli MG, Colvin R, Macones GA, Cahill AG. Neonatal morbidity associated with shoulder dystocia maneuvers. Am J Obstet Gynecol. 2015;212(3):353.e1-e5.

24. Iffy L, Varadi V. Cerebral palsy following cutting of the nuchal cord before delivery. Med Law. 1994;13(3-4):323-330.
25. Reid PC, Osuagwu FI. Symphysiotomy in shoulder dystocia. J Obstet Gynaecol. 1999;19(6):664-666.

26. Woods CE. A principle of physics as applicable to shoulder delivery. Am J Obstet Gynecol. 1943;45(5):796-804.

27. Rubin A. Management of shoulder dystocia. JAMA. 1964;189: 835-837.

28. Jacquemier JM. Du volume de la poitrine et des épaules du foetus considéré comme cause de dystocia dans les presentations de l'extrémité céphalique [Fetal chest and shoulder size as a cause of dystocia in cephalic presentations]. Gaz Hébd Méd Chir. 1860;7(661-3):644692-697698. French.

29. Couder L. De la protection du perinée pendant le passage du tronc après la sortie de la tete [Protecting the perineum during delivery of the body after delivery of the head]. Archiv Tocol Gynecol. 1891;17:508-515. French.

30. Mottet N, Bonneaud M, Eckman-Lacroix A, Ramanah R, Riethmuller D. Active delivery of the anterior arm and incidence of second-degree perineal tears: a clinical practice evaluation. BMC Pregnancy Childbirth. 2017;17(1):141

31. Menticoglou SM. A modified technique to deliver the posterior arm in severe shoulder dystocia. Obstet Gynecol. 2006;108(3 Pt 2):755-757.

32. Schramm M. Impacted shoulders - a personal experience. Aust N ZJ Obstet Gynaecol. 1983;23(1):28-31.

33. Hofmeyr GJ, Cluver CA. Posterior axilla sling traction for intractable shoulder dystocia. BJOG. 2009;116(13):1818-1820.

34. Beer E, Mangiante G, Pecorari D. Distocia delle spalle: storia ed attualit. Roma: CIC Edizioni Internazionali; 2006.

35. Schall JP. Mécanique et techniques obstétricales. 3rd ed. Montpellier: Sauramps; 2007.

36. Collin A, Dellis X, Ramanah R, et al. La dystocie vraie des épaules: analyse de 14 cas traités par la manœuvre de Jacquemier [Severe shoulder dystocia: study of 14 cases treated by Jacquemier's maneuver]. J Gynecol Obstet Biol Reprod (Paris). 2008;37(3):283-290. French.

37. Menticoglou SM. Symphysiotomy for the trapped aftercoming parts of the breech: a review of the literature and a plea for its use. Aust NZ J Obstet Gynaecol. 1990;30(1):1-9.

38. Goodwin TM, Banks E, Millar LK, Phelan JP. Catastrophic shoulder dystocia and emergency symphysiotomy. Am J Obstet Gynecol. 1997; 177(2):463-464.

39. Mola GD. Symphysiotomy: technique, problems and pitfalls, and how to avoid them. PNG Med J. 1995;38(3):231-238.

40. Hope P, Breslin S, Lamont L, et al. Fatal shoulder dystocia: a review of 56 cases reported to the confidential enquiry into stillbirths and deaths in infancy. Br J Obstet Gynaecol. 1998;105(12):1256-1261.

41. Menticoglou S, Schneider C. Resuscitating the baby after shoulder dystocia. Case Rep Obstet Gynecol. 2016;2016:8674167.
International Journal of Women's Health

\section{Publish your work in this journal}

The International Journal of Women's Health is an international, peerreviewed open-access journal publishing original research, reports, editorials, reviews and commentaries on all aspects of women's healthcare including gynecology, obstetrics, and breast cancer. The manuscript management system is completely online and includes

\section{Dovepress}

a very quick and fair peer-review system, which is all easy to use Visit http://www.dovepress.com/testimonials.php to read real quotes from published authors. 